\title{
Human Very Small Embryonic-Like Stem Cells Are Present in Normal Peripheral Blood of Young, Middle-Aged, and Aged Subjects
}

\author{
Hanna Sovalat, Maurice Scrofani, Antoinette Eidenschenk, and Philippe Hénon \\ Institut de Recherche en Hématologie et Transplantation, Hôpital du Hasenrain, 87 avenue d'Altkirch, 68100 Mulhouse, France \\ Correspondence should be addressed to Hanna Sovalat; hsovalat@yahoo.fr
}

Received 17 February 2015; Accepted 27 March 2015

Academic Editor: Mariusz Z. Ratajczak

Copyright (c) 2016 Hanna Sovalat et al. This is an open access article distributed under the Creative Commons Attribution License, which permits unrestricted use, distribution, and reproduction in any medium, provided the original work is properly cited.

\begin{abstract}
The purpose of our study was to determine whether the number of human very small embryonic-like stem cells (huVSELs) would vary depending on the age of humans. HuVSELs frequency was evaluated into the steady-state (SS) peripheral blood (PB) of healthy volunteers using flow cytometry analysis. Their numbers were compared with volunteers' age. Blood samples were withdrawn from 28 volunteers (age ranging from 20 to 70 years), who were distributed among three groups of age: "young" (mean age, 27.8 years), "middle" (mean age, 49 years), and "older" (mean age, 64.2 years). Comparing the three groups, we did not observe any statistically significant difference in huVSELs numbers between them. The difference in mRNA expression for PSC markers as SSEA-4, Oct-4, Nanog, and Sox 2 between the three groups of age was not statistically significant. A similar frequency of huVSELs into the SS-PB of young, middle-aged, and aged subjects may indicate that the VSELs pool persists all along the life as a reserve for tissue repair in case of minor injury and that there is a continuous efflux of these cells from the BM into the PB.
\end{abstract}

\section{Introduction}

Under steady-state conditions, small amounts of hematopoietic stem cells (HSCs) constantly leave the bone marrow $(\mathrm{BM})$, penetrate the tissues, and return to the $\mathrm{BM}$ or peripheral niches via the blood or the lymphatic system [1-3]. Thus, the peripheral blood $(\mathrm{PB})$ may be envisioned as a highway by which HSC can relocate between distant stem cell niches to keep the pool of BM stem cells in balance. In adult organisms, circulating stem/progenitor cells show a circadian rhythm with a peak occurring early in the morning and a nadir at night. Their number increases modestly during minor hematopoietic stresses, such as infection or strenuous exercise, [4-6]. In addition to HSCs, some rare other stem/progenitor cells such as mesenchymal stem cells (MSCs) [7], fibrocytes [8,9], skeletal progenitors [10, 11], and endothelial progenitor cells (EPCs) [12-14] may also circulate into the $\mathrm{PB}$ after tissue/organ injury. Recently, a population of very small embryonic-like stem cells (VSELs) was identified in murine adult bone marrow [15]. Murine VSELs (muVSELs) are small, nonhematopoietic cells with high nuclear/cytoplasm ratio and unorganized euchromatin and express markers of pluripotent embryonic and primordial germ cells. MuVSELs have been identified in most tissues [16]. These cells circulate in a very few number into the PB under steady-state (SS) conditions and in much larger numbers after administration of granulocyte-colony stimulating factor (G-CSF) [17]. It was demonstrated that, either under steady-state conditions or after response to GCSF, circulating VSELs numbers were lower in older animals than in younger ones $[18,19]$. Human VSELs (huVSELs) have been firstly identified in umbilical cord blood as CXCR4 ${ }^{+}$, $\mathrm{CD} 34^{+}, \mathrm{CD}_{33} 3^{+}, \mathrm{Lin}^{-}$, and $\mathrm{CD} 45^{-}$cells enriched for Oct4 and SSEA-4 [20]. We have recently shown that a similar population of huVSELs was present in both adult BM and PB and could be harvested by leukapheresis after G-CSF administration [21].

However, whether the number of VSELs would also vary in humans depending on the age has to be determined. In the present study, we have both assessed the presence of huVSELs into the steady-state $\mathrm{PB}$ of healthy volunteers and compared their numbers in function of age. 


\section{Material and Methods}

2.1. Human Healthy Volunteers. 28 healthy volunteers (12 females and 16 males; mean age, $41.9 \pm 15.4$ years; range $20-70$ years), not taking any medication, were enrolled in this study after informed consent.

$20 \mathrm{~mL}$ of SS-PB was withdrawn by venous puncture from each subject early in the morning to avoid the impact of any physical effort on PB cell counts, collected on EDTA, and immediately processed. The absolute number of white blood cells (WBC) was determined at the same time using a Coulter $\mathrm{A}^{\mathrm{c}} \mathrm{T}$ diff cell counter (Beckman Coulter, Roissy, France).

2.2. Flow Cytometry (FCM) Analysis. Staining and FCM analysis were performed as previously described [21]. Briefly, samples of whole $\mathrm{PB}$ were lysed in hypotonic ammonium chloride buffer (IOTest lysing solution, Beckman Coulter, Roissy, France) to remove red blood cells. Total nucleated cells (TNC) were stained with a mixture of lineages (Lin) associating monoclonal antibodies (MoAbs) conjugated with fluorescein isothiocyanate (FITC). At the same time, phycoerythrin (PE) conjugated-CD45 MoAb clone J33 (Beckman Coulter, Roissy, France) and a combination of allophycocyanin (APC) conjugated MoAbs, CD133 clone AC133 (Miltenyi Biotec, Paris, France), CD34 clone 8G12, or CD184 (CXCR4) clone 12G5 (BD, Le Pont de Claix, France), were added for 30 minutes on ice. Cells were then washed and fixed with $4 \%$ formaldehyde (FA) for 20 minutes. Finally, 7aminoactinomycin D (7-AAD; BD, Le Pont de Claix, France) was added to stain nucleated cells.

FCM analyses were performed using a FACSVantage DIVA fluorescence-activated cell sorting device (BD Biosciences, Erembodegem, Belgium). At least $10^{6}$ small events ranging from 2 to $10 \mu \mathrm{m}$ were included in the gate after comparison with five different size beads calibrated at standard diameters of $1,2,4,6$, and $10 \mu \mathrm{m}$ (Flow Cytometry Size Calibration, Invitrogen/ThermoFischer Scientific, Illkirch, France). CXCR4 ${ }^{+} \mathrm{Lin}^{-} \mathrm{CD} 45^{-}, \mathrm{CD} 34^{+} \mathrm{Lin}^{-} \mathrm{CD} 45^{-}$, or $\mathrm{CD}_{133^{+}} \mathrm{Lin}^{-} \mathrm{CD} 45^{-}$cell subset amounts were counted among the nucleated $7-\mathrm{AAD}^{+}$cells.

Cell subpopulations absolute numbers were calculated in $1 \mathrm{~mL}$ of $\mathrm{PB}$.

2.3. Reverse Transcription-Quantitative-Polymerase Chain Reaction (RT-qPCR) Analysis. RNA extraction from total PBNC and analysis of Oct-4, Nanog, and Sox 2 mRNA were carried out as previously described [21]. Primer sequences of Oct-4, Nanog, Sox2, and $\beta_{2}$ are summarized in Table 1. RNA isolated from H9 and HUES 3 hESC lines [22, 23], kindly provided by the "Plateforme Cellules Souches Embryonnaires Humaines” (Inserm U602 Villejuif, France), was used as reference sample for each $\mathrm{PCR}$ reaction.

2.4. Immunofluorescence Staining. The expression of pluripotency antigens was determined for each healthy volunteer. NC were stained for 2 hours with antibodies against SSEA-4 (clone MC-813-70, mouse monoclonal IgG), Tumor Rejection Antigen (TRA-1-81, clone TRA-1-81, mouse monoclonal IgM), OCT-4A/4B (clone 9E3.2, mouse monoclonal
IgG), and NANOG (goat polyclonal) (Millipore, Molsheim, France), as previously described in detail [21]. Appropriate secondary FITC or tetramethylrhodamine-5-isothiocyanate (TRITC) goat anti-mouse IgG or IgM and FITC-goat antirabbit (Beckman Coulter, Marseille, France) were added for $1 \mathrm{~h}$. The nuclei were labelled with $4^{\prime}, 6$-Diamidino-2phenylindole (DAPI) complemented with Vectashield (Vector Laboratories, Abcys, Paris, France). Cells stained with secondary antibodies only were used as negative controls. Slides with $\mathrm{H} 9$ and/or HUES3 hESC lines were stained similarly and used as positive controls. Fluorescence images were recorded with the AxioVision 4.7 "Full support" system attached to a fluorescent microscope Axiostar Plus Zeiss and captured by AxioCam ICC 1 R3 Cameras (Lordil, Villers-lesNancy, France).

2.5. Statistical Analysis. Data were expressed as mean \pm standard deviation (SD). To verify the difference in expression of each marker between the different age groups, normality data were tested by the Shapiro test. Once normality is verified, one-way ANOVA was chosen to carry out this comparison; on the contrary, the Kruskal-Wallis test was chosen when normality was not verified. $t$-test pairwise analysis was performed when previous tests were significant.

\section{Results}

3.1. VSELs Numbers Do Not Decrease with Aging. Enumeration of circulating VSELs requires FACS-unique gating strategies to focus on very rare and small events (Figures 1(a) and $1(\mathrm{~b}))$.

We applied here the identification strategy which was published in detail by Zuba-Surma and Ratajczak [24]. Three different subpopulations of huVSELs (diameter ranging from 3 to $6 \mu \mathrm{m}$ ) have thus been detected into SS-PB but in very low numbers. Considering altogether the healthy adult volunteers enrolled in this study, $290 \pm 150 \mathrm{CD}^{2} 33^{+} \mathrm{Lin}^{-} \mathrm{CD} 45^{-}$cells, $80 \pm 60 \mathrm{CD}^{2} 4^{+} \mathrm{Lin}^{-} \mathrm{CD} 45^{-}$cells, and $300 \pm 260 \mathrm{CXCR}^{+} \mathrm{Lin}^{-}$ $\mathrm{CD} 45^{-}$cells were counted on average per $\mathrm{mL}$ of total blood.

When volunteers were distributed among three groups of age, "young" (mean age, 27.8 years, range from 20 to 39 years; $n=13$ ), "middle," (mean age, 49 years, range from 40 to 59 years; $n=10$ ), and "older" (mean age, 64.2 years, range from 60 to 70 years; $n=5$ ) groups, not any statistically significant difference in huVSELs frequency (expressed as number of cells $/ \mathrm{mL}$ ) in $\mathrm{PB}$ was observed between these three groups $(P>0.05$; one-way analysis of variance (ANOVA)) (Figure 2).

However, in the older group, all VSELs subset numbers were lower in 3 of the subjects compared to the other 2, but this decline was not statistically significant. It may be due to the low number of "old" patients $(n=5)$ compared to the number of "young" and "middle-aged" groups. It is clearly important to increase the number of patients in the "old" group to have a clear data. This low number of aged healthy volunteers is due to our difficulty to find people with advanced age taking any drug. In fact, in our future investigations, by increasing our collaborations with other laboratories we hope to resolve this problem. 

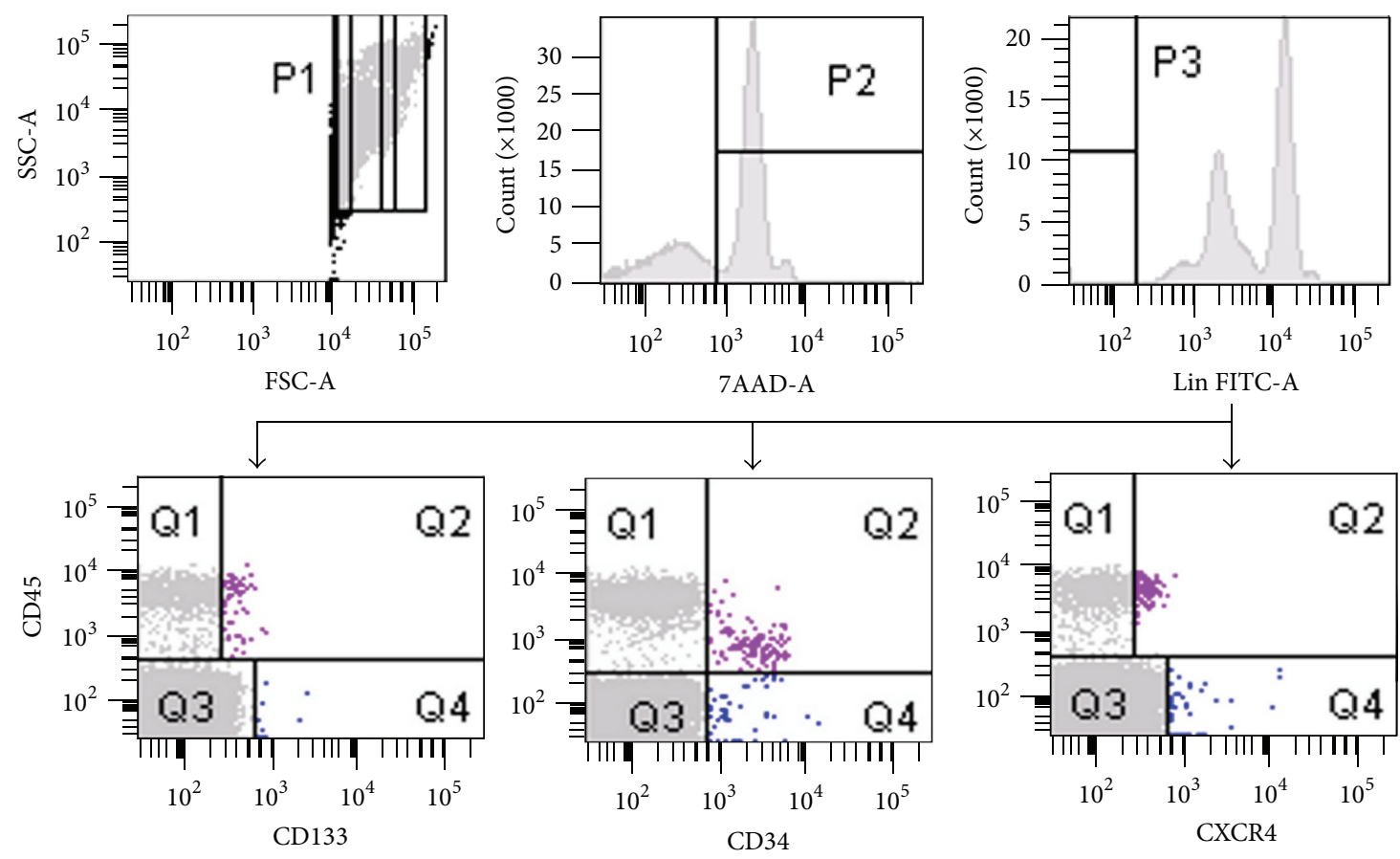

(a)
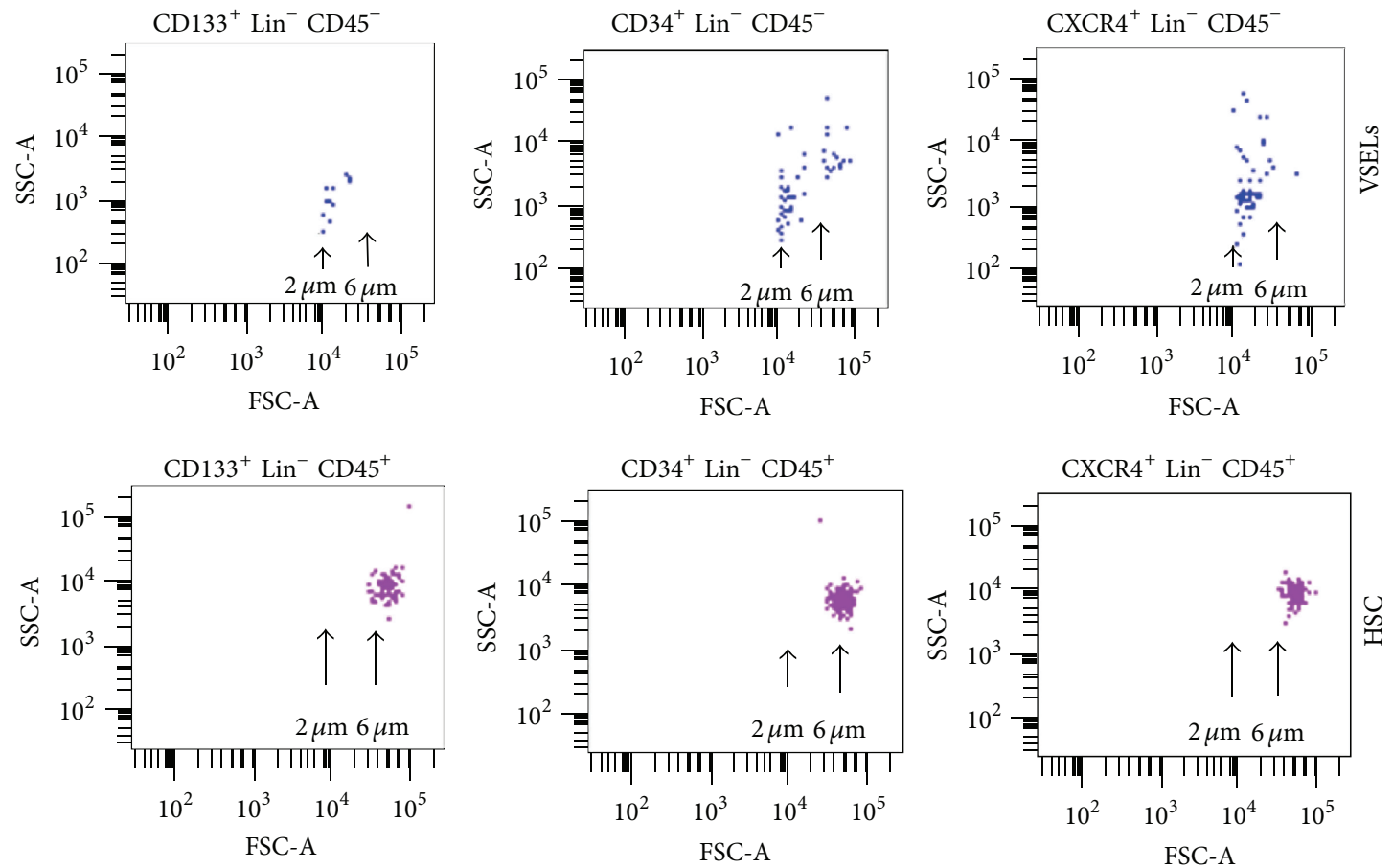

(b)

FIGURE 1: FCM analysis of PB-derived VSELs. Erythrocytes were removed by hypotonic lysis and PB NCs were stained with fluorescein isothiocyanate-lineage markers (Lin), phycoerythrin-CD45 and allophycocyanin-CD133, CD34, or CXCR4 mAbs. (a) Left dot plot: $10^{6}$ events ranging from 2 to $10 \mu \mathrm{m}$ were included in the P2 gate. Right histogram: Lin ${ }^{-}$cells were included in the P1 analysis gate. Middle histogram: $\mathrm{P} 1$ gated cells stained with 7-aminoactinomycin D (7-AAD) were included in the P2 gate. Right histogram: Lin ${ }^{-}$cells were included in the P3 gate and were analyzed for CD45 coexpression with CD133, CD34, or CXCR4 antigens. (b) Dot plots showing the size of analyzed CD133 ${ }^{+}$, $\mathrm{CD} 34^{+}$, or CXCR4 ${ }^{+}, \mathrm{Lin}^{-}, \mathrm{CD} 45^{-}$VSELs (upper row) and CD133 ${ }^{+}, \mathrm{CD} 34^{+}$, or CXCR4 $4^{+}, \mathrm{Lin}^{-}, \mathrm{CD} 45^{+}$HSC (lower row). 
TABLE 1: Sequences of the forward and reverse primers employed for qPCR.

\begin{tabular}{lcc}
\hline & \multicolumn{1}{c}{ Forward } & Reverse \\
\hline Oct-4 & GAT GTG GTC CGA GTG TGG TTC T & TGT GCA TAG TCG CTG CTT GAT \\
Nanog & GCA GAA GGC CTC AGC ACC TA & AGG TTC CCA GTC GGG TTC A \\
Sox2 & TAC AGC ATG TCC TAC TCG CAG & GAG GAA GAG GTA ACC ACA GGG \\
Beta-2-microglobuline & AAT GCG GCA TCT TCA AAC CT & TGA CTT TGT CAC AGC CCA AGA TA \\
\hline
\end{tabular}

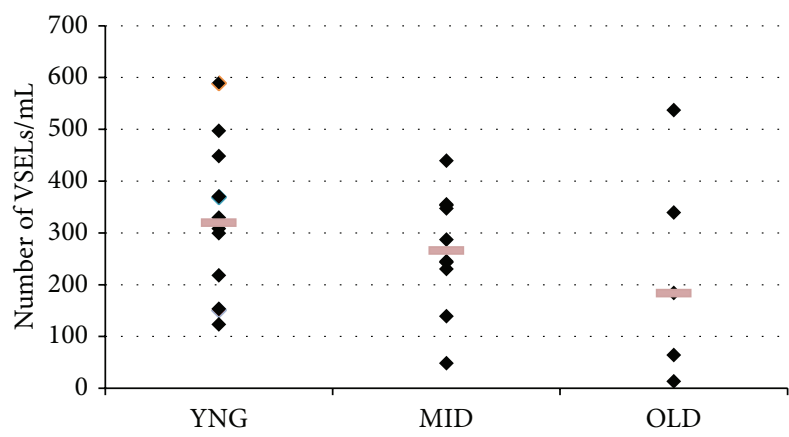

(a)

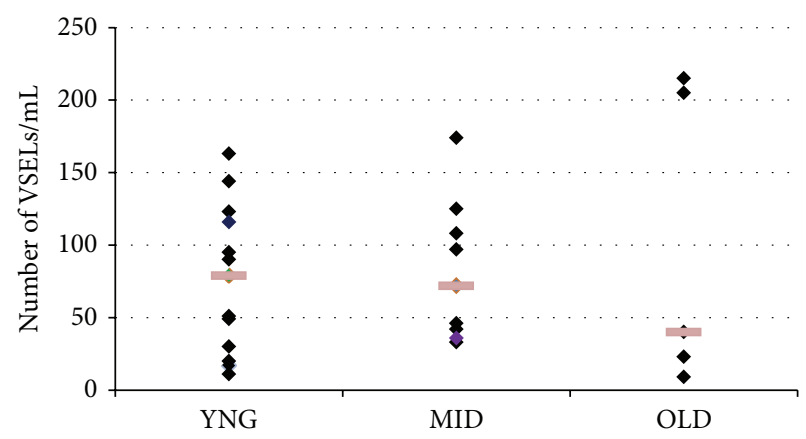

(b)

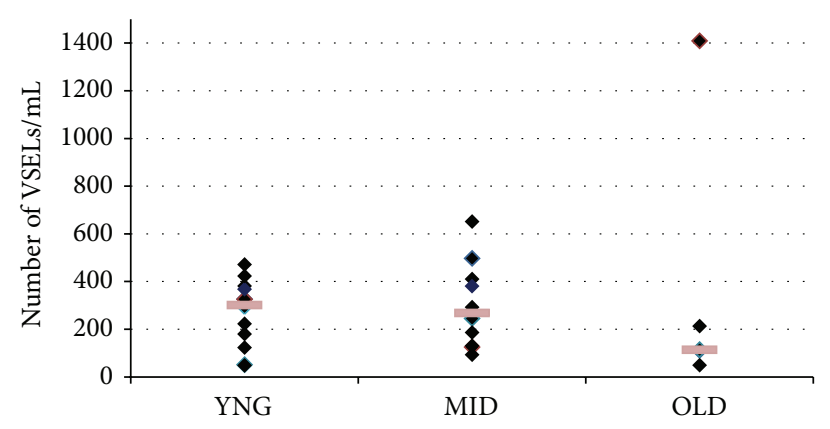

(c)

Figure 2: Age-dependent frequency of $\operatorname{Lin}^{-} / \mathrm{CD} 45^{-}$cell subsets expressing (a) CD133, (b) CD34, and (c) CXCR4 into the PB. Three groups of healthy human volunteers were designed according to their age: "young" (20-39 years; $n=13)$, "middle" ( $40-59$ years; $n=10)$, and "older" $(60-70$ years; $n=5)$. Frequency of Lin $^{-}$CD $45^{-}$cell subsets was calculated per $\mathrm{mL}$ of PB. Each square represents the number of VSELs/mL per volunteer in each group. The difference between the three groups of volunteers is not statistically significant $(P>0.05$; one-way analysis of variance (ANOVA)).

3.2. A Population of Small CD45 Cells Expressing Several Pluripotent Stem Cell Markers Is Present in SS-PB of Young, Middle, and Older Subjects. Immunofluorescence staining showed that $\mathrm{CD} 45^{-}$cell subsets express both specific pluripotent stem cell (PSC) markers such as SSEA-4 and TRA-1-81 on their surface and OCT- 4 and NANOG transcription factors at the protein level (Figure 3).

Expression of PSC markers was confirmed by RT-qPCR. The differences in mRNA expression for those markers between the three groups of age were not statistically significant $(P>0.05$, Kruskal-Wallis test) (Figure 4$)$.

\section{Discussion}

The PB could be envisioned as a highway by which stem cells are trafficking in the body to keep in balance a pool of stem cells located in different niches in peripheral tissues. In this context, the BM has been proposed to be a main reservoir for these circulating cells [25]. In addition to HSCs, several other types of stem/progenitor cells have been described in the adult BM, such as mesenchymal stem cells (MSCs) [26], marrow-isolated adult multilineage inducible (MIAMI) cells [27], and multipotent adult stem cells (MASCs) [28]. A population of VSELs was first identified in the murine BM by the group of Ratajczak [15]. These cells express several markers characteristics for embryonic stem cells (ESCs), such as Oct-4, Nanog, and SSEA-1. MuVSELs circulate at very low numbers $(\sim 150-300 / \mathrm{mL})$ into the murine SS-PB. Wojakowski et al. have also recently shown that very small numbers of VSELs ( $80-1300$ cells/mL) could be detected under steadystate condition into the SS-PB of healthy humans, aged from 30 to 50 years, thus reflecting a continuous efflux of these cells from the BM [29]. Our present study, performed in a population of healthy adults with a larger age range (from 20 to 70 years), confirms that huVSELs actually circulate at very low levels (80-300/mL) into the SS-PB, in young and in older 

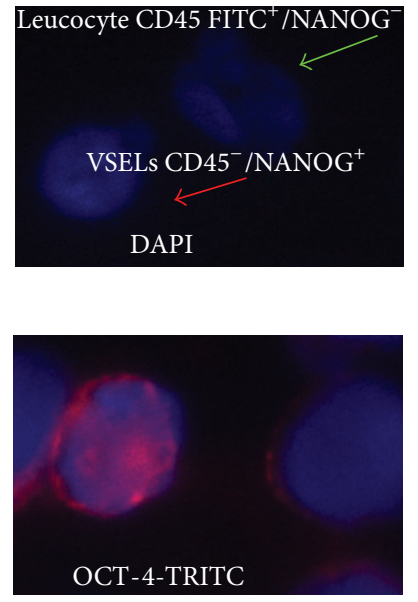
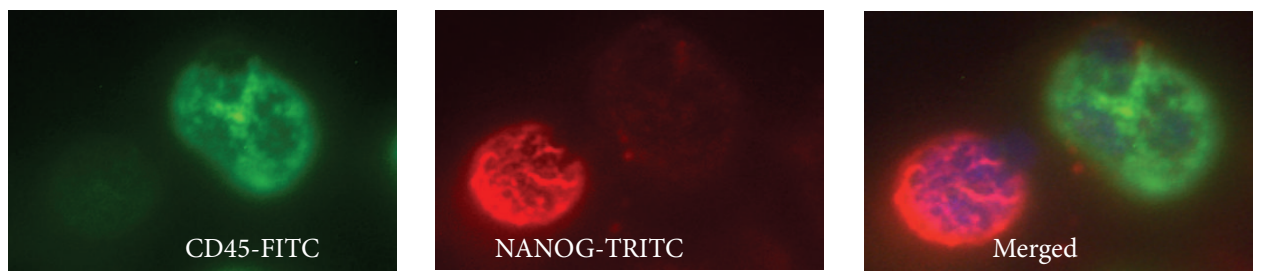

(a)
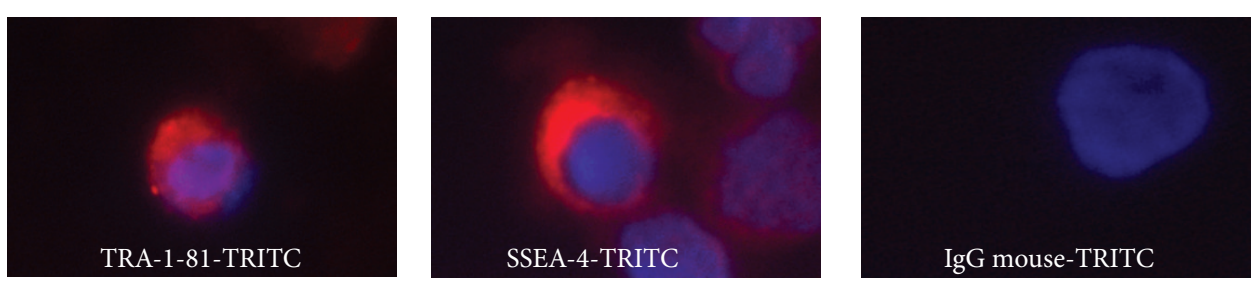

(b)

FIGURE 3: Immunofluorescence analysis of PB-derived VSELs. A typical triple-staining with $4^{\prime}$,6-diamidino-2-phenylindole (DAPI) (blue: nuclei), fluorescein isothiocyanate- (FITC-) CD45 (green fluorescence), and tetramethylrhodamine-5-isothiocyanate- (TRITC-) SSEA- ${ }^{-}$, TRA-1-81, OCT-4, or NANOG (red) shows (a) VSELs: small CD45 cells NANOG ${ }^{+}$and leukocytes: greater CD $45^{+} \mathrm{NANOG}^{-}$cells and (b) VSELs: CD $45^{-}$cells which express OCT-4 in nuclei or TRA-1-81 and SSEA-4 on the surface.

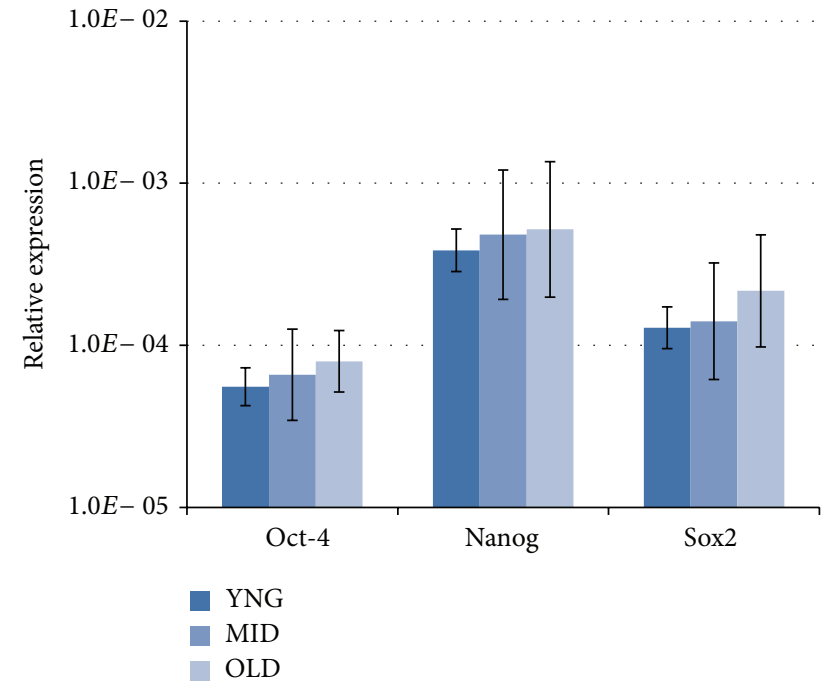

FIGURE 4: Relative expressions of PSC markers, Oct-4, Nanog, and Sox2, were measured by RT-qPCR and compared using equal amounts of mRNA isolated from CNT PB of young, middle, and older healthy volunteers. The relative expression of each PSC marker is calculated according to a positive control PCR (RNA isolated from H9 and HUES 3 hESC lines). Data represent the mean \pm standard deviation for each age group. The difference in mRNA expression of these markers between the three groups of age was not statistically significant $(P>0.05$, Kruskal-Wallis test).

subjects. Furthermore, they can be physiologically mobilized in order to participate in tissue/organ repair. Indeed, in several pathological situations, both in mice and in humans (e.g., heart infarct, stroke, skin burn injury, Crohn's disease, etc.), VSELs are released into the $\mathrm{PB}$ and their circulating numbers thus significantly increase [30-34]. However, when VSELs are released from the BM as a physiological response to tissue/organ injuries, even if they are able to home to the damaged areas, it is likely that they can only participate in the regeneration of minor tissue injuries and not of the largest ones, because of their too small amounts. To improve their potential regenerative impact, murine and huVSELs could then be mobilized by G-CSF administration [17, 21]. We have thus observed that circulating huVSELs amounts increase up to 2-4-fold after 4 days of G-CSF administration [35].

In case of myocardial infarct, it was also noticed that the intensity of the pic of VSELs spontaneous mobilization correlates with the extend of the ischemic $[36,37]$ or stroke lesion [32]. The knowledge of time occurrence of the mobilization pic might thus allow the determination of a therapeutic "window" useful for clinical application. On the other hand, the spontaneous (equal to physiological) mobilization rate could reflect the overall "regenerative potential" of an adult organism. Reduced spontaneous stem cell mobilization has been associated with poor prognosis in patients with myocardial infarction [37]. Thus, we suggest that the mean steady-state level of circulating huVSELs determined from a large enough cohort of healthy subjects might serve as a reference value which could be used as a baseline from which the physiopathological VSEL mobilization rate following an organ injury (e.g., heart infarct or stroke) would be correlated with its severity and its prognosis.

We also focused our study on the potential effect of aging on the amounts of huVSELs circulating in PB under steadystate conditions. The effects of aging on the HSC compartment have been extensively studied in mice. In the murine HSC system, several studies have reported an increase in the absolute number of phenotypically defined HSCs, although elderly animals have a reduced repopulation potential when compared to younger organisms [38-42]. Additionally, other investigators interestingly showed in mice that the frequency of the HSC subpopulation phenotypically defined as lineage 
negative Sca $-1^{+}, \mathrm{c}^{-\mathrm{kit}^{+}}$, Thy- $1^{+}, \mathrm{CD} 135^{-}$side population (SP) steadily increased in the $\mathrm{BM}$ of the femurs and tibias with age. But, although long-term repopulation assays indicated that SP cells are still present and capable of self-renewal and differentiation in older mice, they have shown a lower homing efficiency than those from younger mice [43].

A little study on age-related changes in HSC number and function has been reported in humans. Although indirectly demonstrated through clinical data, there is evidence that human HSC function declines with age. For example, hematopoietic engraftment following HSCs transplantation is often faster and better sustained when the donor (in the autologous as well in the allogeneic setting) is young. Also, elderly people frequently and specifically develop hematological disorders due to acquired HSCs abnormalities, as, for example, myelodysplastic syndromes (MDS). Marley et al. have also found clear in vitro correlations between the relative numbers and function of clonogenic myeloid progenitors (CFU-GM) and age of the donor: in vitro colony formation study has indeed revealed that, although the absolute number of colony forming cells increases with age, their individual self-renewal capacity decreases [44].

Regarding VSELs, it was recently demonstrated in steadystate mice that, unlike HSC and SP, the number of muVSELs in the $\mathrm{BM}$ and the $\mathrm{PB}$ gradually decreases with age, in parallel with their ability to form VSEL-derived spheres (VSEL-DS) containing primitive stem cells, which may explain why the regenerative processes are less efficient in advanced age [18, 19]. Moreover, the number of VSELs is much higher in the BM of long-lived (C57BL/6) as compared to short-lived (DBA/2) mice [15], which allows supposing a positive correlation between high VSEL numbers and a greater length of life.

The effects of aging on the VSELs compartment had never been evaluated in humans until our present study. In response to G-CSF administration, we did not observe agedependent huVSELs release into leukapheresis products from adult cancer patients aged from 34 to 71 years [35]. Here, we interestingly show for the first time that the numbers of circulating huVSELs in healthy subjects aged from 20 to 70 years were not statistically different whichever the age bracket is and thus would not seem to decline significantly with age. However, as we observed that the number of the all VSELs subpopulations was lower, while not significantly, in 3 subjects in the older group, this would nevertheless suggest that the frequency of $\mathrm{CD} 133^{+}, \mathrm{CD} 34^{+}$, or CXCR4 $4^{+}$VSELs subpopulations could progressively run out with aging. A study including more number of older subjects is necessary in future to support this hypothesis.

On the other side, it is supposed that, as we age, stem cells likely progressively lose at least a part of their selfrenewal and/or differentiation capabilities, which would lead to reduce their cell tissue regeneration potential and consequently contribute to the global somatic senescence. Thus, it would be crucial to demonstrate whether the proliferation potential of huVSELs also depends on age in humans. However, while murine VSELs proliferation and differentiation has been well demonstrated in vitro, we and others had failed to demonstrate such proliferative capacities of huVSEls by in vitro studies up to now. The major difficulty is that these cells stay in dormant state and nobody had already identified the combination of factors necessary to trigger the expansion/differentiation processes in purified huVSELs ex vivo cultures. Hopefully, the Taichman's group has very recently shown that huVSELs are able to generate multiple tissues within an osseous wound in immune-deficient mouse when cocultured with the $\mathrm{C} 2 \mathrm{C} 12$ cell line [45], thus demonstrating for the very first time in vitro that huVSELs have actually the capacity to self-renew.

\section{Conclusions}

In this study, we demonstrate that huVSELs circulate at a similar frequency into the SS-PB of young, middle-aged, and aged subjects. This may indicate that the pool of VSELs persists along the life as a reserve for tissue repair in case of minor injury, and that there is a continuous efflux of these cells from the $\mathrm{BM}$ into the $\mathrm{PB}$. We hypothesize that in case of major health complications (e.g., heart infarct, stroke) circulating VSELs could be isolated even from elderly patients, ex vivo expanded, and reinjected back into the same recipients for therapeutic purposes. Of course, such a strategy should be first tested in animal models. First and foremost, the actual VSELs' ability to self-renew and to differentiate has to be determined before they could be used for cell-based clinical therapies. In the recent study, the Taichman's group now opens the way for future huVSEL-based regenerative therapies at least for osseous, neural, and connective tissue disorders.

\section{Conflict of Interests}

The authors declare that there is no conflict of interests regarding the publication of this paper.

\section{Acknowledgments}

This work was supported by a grant from the REUNICA Group (Mulhouse, France). The authors thank A. Merlin from CellProthera SAS, Mulhouse, France, for statistical consultation.

\section{References}

[1] J.-P. Lévesque, I. G. Winkler, S. R. Larsen, and J. E. J. Rasko, "Mobilization of bone marrow-derived progenitors," in Bone Marrow-Derived Progenitors, vol. 180 of Handbook of Experimental Pharmacology, pp. 3-36, Springer, Berlin, Germany, 2007.

[2] P. J. Quesenberry, G. Colvin, G. Dooner, M. Dooner, J. M. Aliotta, and K. Johnson, "The stem cell continuum: cell cycle, injury, and phenotype lability," Annals of the New York Academy of Sciences, vol. 1106, pp. 20-29, 2007.

[3] S. Massberg and U. H. von Andrian, "Novel trafficking routes for hematopoietic stem and progenitor cells," Annals of the New York Academy of Sciences, vol. 1176, pp. 87-93, 2009.

[4] B. Bittira, D. Shum-Tim, A. Al-Khaldi, and R. C.-J. Chiu, "Mobilization and homing of bone marrow stromal cells in 
myocardial infarction," European Journal of Cardio-Thoracic Surgery, vol. 24, no. 3, pp. 393-398, 2003.

[5] A. M. Leone, S. Rutella, G. Bonanno et al., "Mobilization of bone marrow-derived stem cells after myocardial infarction and left ventricular function," European Heart Journal, vol. 26, no. 12, pp. 1196-1204, 2005.

[6] D. C. Hess and C. V. Borlongan, "Stem cells and neurological diseases," Cell Proliferation, vol. 41, supplement 1, pp. 94-114, 2008.

[7] G. Y. Rochefort, B. Delorme, A. Lopez et al., "Multipotential mesenchymal stem cells are mobilized into peripheral blood by hypoxia," Stem Cells, vol. 24, no. 10, pp. 2202-2208, 2006.

[8] R. Bucala, L. A. Spiegel, J. Chesney, M. Hogan, and A. Cerami, "Circulating fibrocytes define a new leukocyte subpopulation that mediates tissue repair," Molecular Medicine, vol. 1, no. 1, pp. 71-81, 1994.

[9] R. J. Phillips, M. D. Burdick, K. Hong et al., "Circulating fibrocytes traffic to the lungs in response to CXCL12 and mediate fibrosis," Journal of Clinical Investigation, vol. 114, no. 3, pp. 438-446, 2004.

[10] M. A. Long, S. Y. Corbel, and F. M. V. Rossi, "Circulating myogenic progenitors and muscle repair," Seminars in Cell and Developmental Biology, vol. 16, no. 4-5, pp. 632-640, 2005.

[11] S. A. Kuznetsov, M. H. Mankani, S. Gronthos, K. Satomura, P. Bianco, and P. G. Robey, "Circulating skeletal stem cells," The Journal of Cell Biology, vol. 153, no. 5, pp. 1133-1140, 2001.

[12] Q. Shi, S. Rafii, M. H. Wu et al., "Evidence for circulating bone marrow-derived endothelial cells," Blood, vol. 92, no. 2, pp. 362367, 1998.

[13] S. Rafii, "Circulating endothelial precursors: mystery, reality, and promise," The Journal of Clinical Investigation, vol. 105, no. 1, pp. 17-19, 2000.

[14] F. Dignat-George, A. Blann, and J. Sampol, "Circulating endothelial cells in acute coronary syndromes," Blood, vol. 95, no. 2, p. 728, 2000.

[15] M. Kucia, R. Reca, F. R. Campbell et al., "A population of very small embryonic-like (VSEL) CXCR4(+)SSEA-1(+)Oct-4+ stem cells identified in adult bone marrow," Leukemia, vol. 20, no. 5, pp. 857-869, 2006.

[16] M. Z. Ratajczak, B. Machalinski, W. Wojakowski, J. Ratajczak, and M. Kucia, "A hypothesis for an embryonic origin of pluripotent Oct-4(+) stem cells in adult bone marrow and other tissues," Leukemia, vol. 21, no. 5, pp. 860-867, 2007.

[17] M. J. Kucia, M. Wysoczynski, W. Wu, E. K. Zuba-Surma, J. Ratajczak, and M. Z. Ratajczak, "Evidence that very small embryonic-like stem cells are mobilized into peripheral blood," Stem Cells, vol. 26, no. 8, pp. 2083-2092, 2008.

[18] M. Z. Ratajczak, E. K. Zuba-Surma, D.-M. Shin, J. Ratajczak, and M. Kucia, "Very small embryonic-like (VSEL) stem cells in adult organs and their potential role in rejuvenation of tissues and longevity," Experimental Gerontology, vol. 43, no. 11, pp. 1009-1017, 2008.

[19] E. K. Zuba-Surma, W. Wu, J. Ratajczak, M. Kucia, and M. Z. Ratajczak, "Very small embryonic-like stem cells in adult tissues-Potential implications for aging," Mechanisms of Ageing and Development, vol. 130, no. 1-2, pp. 58-66, 2009.

[20] M. Kucia, M. Halasa, M. Wysoczynski et al., "Morphological and molecular characterization of novel population of CXCR $4^{+}$ SSEA $-4^{+}$Oct- $4^{+}$very small embryonic-like cells purified from human cord blood-preliminary report," Leukemia, vol. 21, no. 2, pp. 297-303, 2007.
[21] H. Sovalat, M. Scrofani, A. Eidenschenk, S. Pasquet, V. Rimelen, and P. Hénon, "Identification and isolation from either adult human bone marrow or G-CSF-mobilized peripheral blood of $\mathrm{CD} 4^{+} / \mathrm{CD} 33^{+} / \mathrm{CXCR}^{+} / \mathrm{Lin}^{-} \mathrm{CD} 45^{-}$cells, featuring morphological, molecular, and phenotypic characteristics of very small embryonic-like (VSEL) stem cells," Experimental Hematology, vol. 39, no. 4, pp. 495-505, 2011.

[22] C. A. Cowan, I. Klimanskaya, J. McMahon et al., "Derivation of embryonic stem-cell lines from human blastocysts," The New England Journal of Medicine, vol. 350, no. 13, pp. 1353-1356, 2004.

[23] J. A. Thomson, J. Itskovitz-Eldor, S. S. Shapiro et al., "Embryonic stem cell lines derived from human blastocysts," Science, vol. 282, no. 5391, pp. 1145-1147, 1998.

[24] E. K. Zuba-Surma and M. Z. Ratajczak, "Unit 9.29. Overview of very small embryonic-like stem cells (VSELs) and methodology of their identification and isolation by flow cytometric methods," in Current Protocols in Cytometry, J. P. Robinson, Ed., chapter 9, 2010.

[25] T. Papayannopoulou, "Bone marrow homing: the players, the playfield, and their evolving roles," Current Opinion in Hematology, vol. 10, no. 3, pp. 214-219, 2003.

[26] Y. Jiang, B. N. Jahagirdar, R. L. Reinhardt et al., "Pluripotency of mesenchymal stem cells derived from adult marrow," Nature, vol. 418, no. 6893, pp. 41-49, 2002.

[27] G. D'Ippolito, G. A. Howard, B. A. Roos, and P. C. Schiller, "Isolation and characterization of marrow-isolated adult multilineage inducible (MIAMI) cells," Experimental Hematology, vol. 34, no. 11, pp. 1608-1610, 2006.

[28] Y. Jiang, B. Vaessen, T. Lenvik, M. Blackstad, M. Reyes, and C. M. Verfaillie, "Multipotent progenitor cells can be isolated from postnatal murine bone marrow, muscle, and brain," Experimental Hematology, vol. 30, no. 8, pp. 896-904, 2002.

[29] W. Wojakowski, M. Kucia, R. Liu et al., "Circulating very small embryonic-like stem cells in cardiovascular disease," Journal of Cardiovascular Translational Research, vol. 4, no. 2, pp. 138-144, 2011.

[30] W. Wojakowski, M. Tendera, M. Kucia et al., "Mobilization of bone marrow-derived Oct $-4^{+}$SSEA $-4^{+}$very small embryoniclike stem cells in patients with acute myocardial infarction," Journal of the American College of Cardiology, vol. 53, no. 1, pp. $1-9,2009$.

[31] E. K. Zuba-Surma, M. Kucia, B. Dawn, Y. Guo, M. Z. Ratajczak, and R. Bolli, "Bone marrow-derived pluripotent very small embryonic-like stem cells (VSELs) are mobilized after acute myocardial infarction," Journal of Molecular and Cellular Cardiology, vol. 44, no. 5, pp. 865-873, 2008.

[32] E. Paczkowska, M. Kucia, D. Koziarska et al., "Clinical evidence that very small embryonic-like stem cells are mobilized into peripheral blood in patients after stroke," Stroke, vol. 40, no. 4, pp. 1237-1244, 2009.

[33] J. Drukała, E. Paczkowska, M. Kucia et al., "Stem cells, including a population of very small embryonic-like stem cells, are mobilized into peripheral blood in patients after skin burn injury," Stem Cell Reviews and Reports, vol. 8, no. 1, pp. 184-194, 2012.

[34] W. Marlicz, E. Zuba-Surma, M. Kucia, W. Blogowski, T. Starzynska, and M. Z. Ratajczak, "Various types of stem cells, including a population of very small embryonic-like stem cells, are mobilized into peripheral blood in patients with Crohn's disease," Inflammatory Bowel Diseases, vol. 18, no. 9, pp. 17111722, 2012. 
[35] H. Sovalat, M. Scrofani, A. Eidenschenk, M. Ojeda-Uribe, and P. Hénon, "G-CSF Administration induces mobilization of human very small embryonic like (VSEL) stem cells into peripheral blood of cancer patients," Experimental Hematology, vol. 40, no. 8, pp. S59-S60, 2012.

[36] W. Wojakowski, M. Tendera, A. Zebzda et al., "Mobilization of $\mathrm{CD} 34(+), \operatorname{CD} 117(+), \operatorname{CXCR} 4(+)$, c-met(+) stem cells is correlated with left ventricular ejection fraction and plasma NTproBNP levels in patients with acute myocardial infarction," European Heart Journal, vol. 27, no. 3, pp. 283-289, 2006.

[37] A. Abdel-Latif, E. K. Zuba-Surma, K. M. Ziada et al., "Evidence of mobilization of pluripotent stem cells into peripheral blood of patients with myocardial ischemia," Experimental Hematology, vol. 38, no. 12, pp. 1131-1142, 2010.

[38] S. J. Morrison, A. M. Wandycz, K. Akashi, A. Globerson, and I. L. Weissman, "The aging of hematopoietic stem cells," Nature Medicine, vol. 2, no. 9, pp. 1011-1016, 1996.

[39] K. Sudo, H. Ema, Y. Morita, and H. Nakauchi, "Age-associated characteristics of murine hematopoietic stern cells," Journal of Experimental Medicine, vol. 192, no. 9, pp. 1273-1280, 2000.

[40] M. Kim, H.-B. Moon, and G. J. Spangrude, "Major age-related changes of mouse hematopoietic stem/progenitor cells," Annals of the New York Academy of Sciences, vol. 996, pp. 195-208, 2003.

[41] D. E. Harrison, "Long-term erythropoietic repopulating ability of old, young, and fetal stem cells," Journal of Experimental Medicine, vol. 157, no. 5, pp. 1496-1504, 1983.

[42] D. J. Rossi, D. Bryder, J. M. Zahn et al., "Cell intrinsic alterations underlie hematopoietic stem cell aging," Proceedings of the National Academy of Sciences of the United States of America, vol. 102, no. 26, pp. 9194-9199, 2005.

[43] D. J. Pearce, F. Anjos-Afonso, C. M. Ridler, A. Eddaoudi, and D. Bonnet, "Age-dependent increase in side population distribution within hematopoiesis: implications for our understanding of the mechanism of aging," Stem Cells, vol. 25, no. 4, pp. 828835, 2007.

[44] S. B. Marley, J. L. Lewis, R. J. Davidson et al., "Evidence for a continuous decline in haemopoietic cell function from birth: application to evaluating bone marrow failure in children," British Journal of Haematology, vol. 106, no. 1, pp. 162-166, 1999.

[45] A. M. Havens, H. Sun, Y. Shiozawa et al., "Human and murine very small embryonic-like cells represent multipotent tissue progenitors, in vitro and in vivo," Stem Cells and Development, vol. 23, no. 7, pp. 689-701, 2014. 

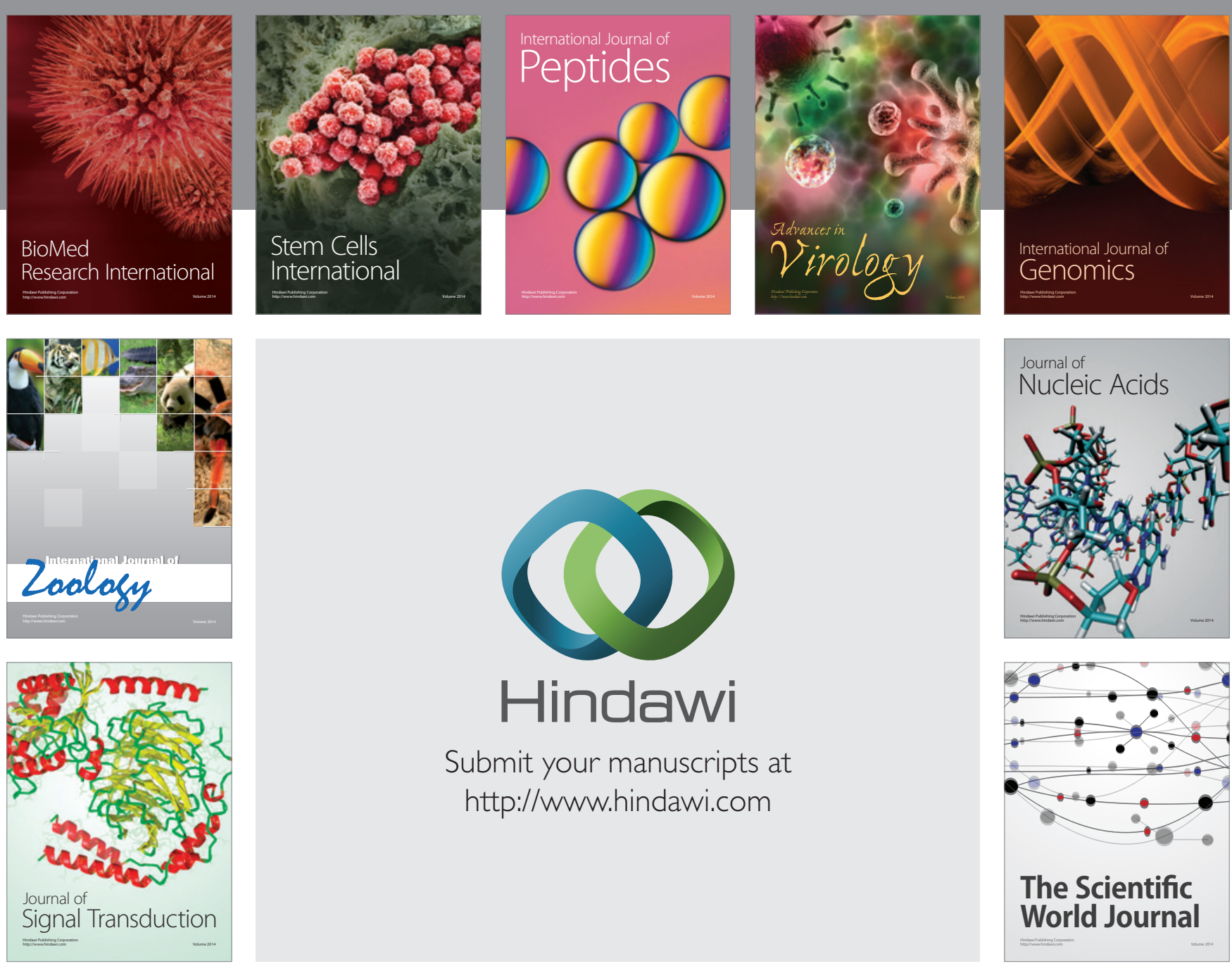

Submit your manuscripts at

http://www.hindawi.com
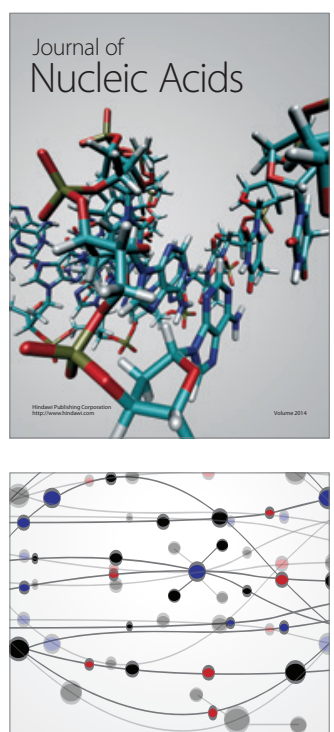

The Scientific World Journal
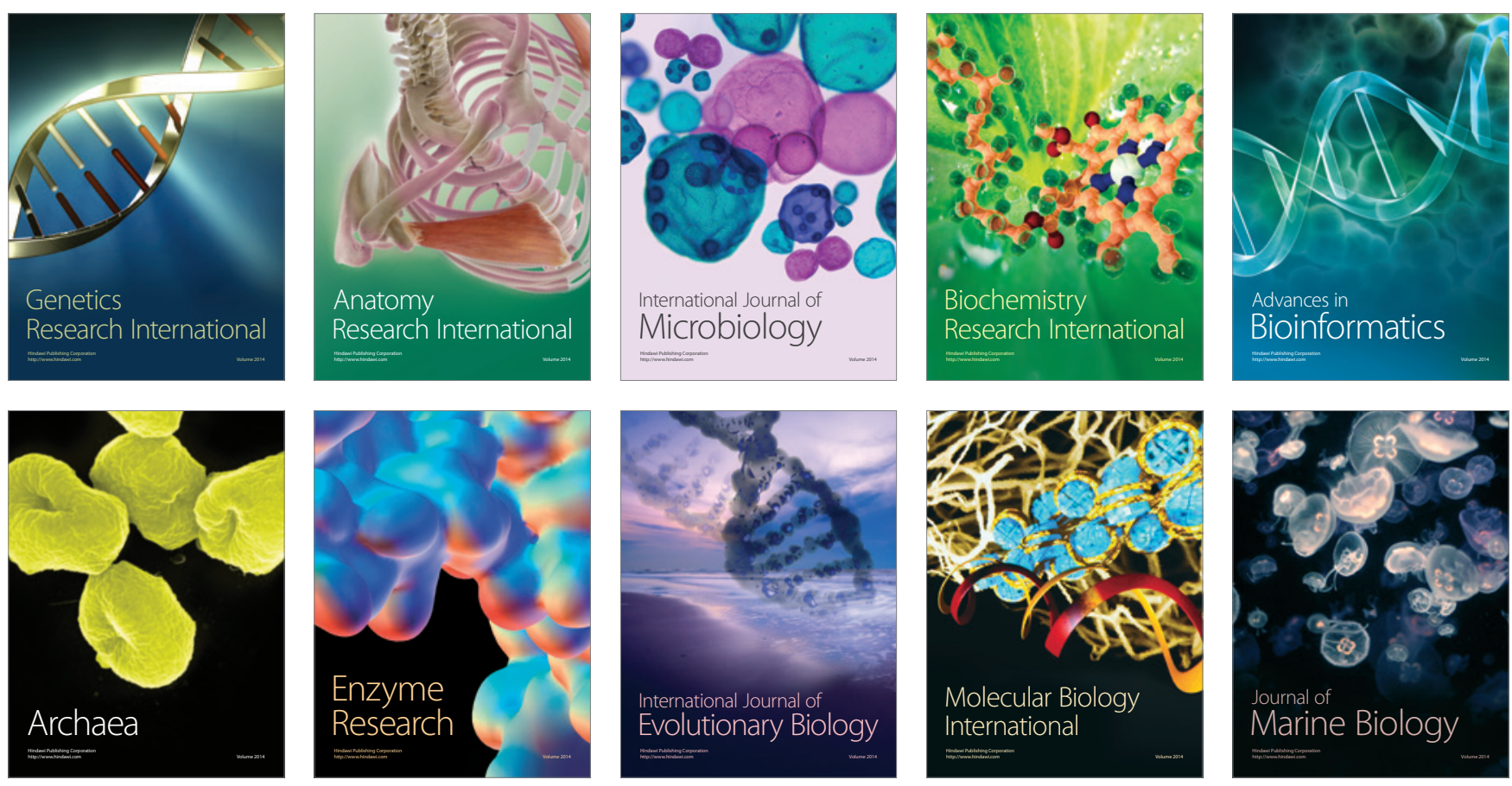